\title{
Diseño para la participación de comunidades e iniciativas en el post-conflicto colombiano: cartografías para diseñar la paz desde los bienes comunes
}

\begin{abstract}
Resumen
En Colombia, a pesar de su constante situación de conflicto armado, existen comunidades que crean paz desde sus acciones cotidianas, desde su relación con los otros y su entorno natural, desde la resistencia no violenta a agentes opresores externos, desde la construcción de sistemas de gobierno y participación que vinculan la diversidad y desde la creación de lazos de confianza que posibilitan agenciar sistemas de articulación social de manera más horizontal o de jerarquías transitorias. Este trabajo de investigación en diseño ${ }^{1}$ presenta un prototipo metodológico para cartografiar iniciativas y comunidades de paz desde la base, que brinde una guía para crear, mantener y potenciar formas no violentas de relaciones sociales. La técnica involucra: El pensamiento en diseño como lugar epistemológico y la creación y mantenimiento de bienes comunes como filosofía. Todo esto para diseñar acciones, espacios y artefactos dentro de las comunidades, con capacidad de fortalecer ambientes sin violencia, partiendo de la hipótesis: Sin bienes comunes no hay paz.
\end{abstract}

Hacia el final, este texto aunque plantea una técnica metodológica que nace de la academia, considera posibilidades futuras en términos de la intervención no academicista con comunidades, e invita a considerar las prácticas simbólicas de éstas para diseñar la paz como bien común.

\author{
Adriana Castrillón Arango \\ Maestría en Diseño y Creación \\ Interactiva \\ Docente de Artes Visuales \\ Universidad del Quindío y directora \\ de la Fundación Visonte. \\ Quindío, Colombia \\ Correo electrónico: adriana. \\ castrillon@visonte.co \\ adriana.castrillon@gmail.com \\ ๑ orcid.org/0000-0001-8575-6736 \\ Google Scholar
}

Recibido: julio 18 de 2018 Aprobado: julio 25 de 2019

\section{Palabras clave:}

Bienes comunes, co-creación, investigación para la paz, pensamiento en diseño, postconflicto.

\footnotetext{
${ }^{1}$ El documento completo de investigación puede consultarse en: https://is.gd/tesis_ pazbienescomunesdiseno
} 


\section{Design for the participation of communities and initiatives in the Colombian post-conflict: cartographies to design peace from the common goods}

\begin{abstract}
Despite the constant situation of armed conflict, there are communities in Colombia that create peace from their daily actions, from their relationship with others, from their natural environment, from non-violent resistance to external oppressive agents, from the construction of systems of government and participation that link diversity and from the creation of bonds of trust that make it possible to organize systems of social articulation in a horizontal way or of transitory hierarchies. This research work in Design presents a methodological prototype for mapping initiatives and communities of peace from the base which provides a guide to create, maintain and enhance non-violent forms of social relations. The technique involves design thinking as an epistemological place and the creation and maintenance of common goods as philosophy. All this to design actions, spaces and artifacts within communities with the capacity to strengthen environments without violence based on the hypothesis that, without common goods, there is no peace. Despite this text proposes a methodological technique that arises from the academy, considers future possibilities in terms of non-academic intervention with communities, and invites to consider their symbolic practices to design peace as a common good.
\end{abstract}

Key words:

The Common goods, design thinking, peace, co-creation, research for peace, post-conflict. 


\title{
Introducción
}

\author{
"Solo los pueblos con \\ convicciones tienen esperanza." \\ Bertolt Brecht
}

Colombia se ha debatido la terminación del conflicto armado interno, entre la solución negociada y la salida militar, con esto, ante la imposibilidad de resolución o transformación del mismo por alguna de las dos vías, en octubre de 2013 se abren nuevamente negociaciones de paz entre el gobierno del Presidente Juan Manuel Santos y el movimiento insurgente FARC-EP ${ }^{1}$. En 2016, tras los diálogos de paz en La Habana, se logra la firma de un acuerdo ${ }^{2}$ con el grupo armado que abre nuevas preguntas, esta vez relacionadas con el alcance de formas pacíficas de accionar social y el mantenimiento en el tiempo de éstas, para que las historias de las violencias pasadas no fueran repetidas.

La complejidad para la superación del conflicto armado colombiano necesita ser asumida desde la dimensión humana (Hernández Delgado, 2014, p. 69) y exige un abordaje integral y dinámico con una mirada que supere lo puramente militar. Con este marco y resaltando que por primera vez Colombia cuenta con voluntad política para superar parte de sus conflictividades violentas e iniciativas sociales que lo respaldan, el documento final del acuerdo de paz resultado de los diálogos de La Habana, marca un primer paso adelante dentro de la trasformación del país.

La pregunta del trabajo de investigación del cual da cuenta este texto fue: ¿Cómo diseñar la paz desde los bienes comunes, con prototipos para la participación ciudadana, en el marco del post-conflicto colombiano?; analizando que el diseño como epistemología, en cuanto busca entender lo complejo, piensa la relación

Sigla de Fuerzas Armadas Revolucionarias Colombianas - Ejército del Pueblo.

${ }^{2}$ El texto completo del acuerdo puede consultarse en: https://is.gd/acuerdo_final_paz 
de lo humano para la generación de propuestas, asume un conocimiento a través de la acción reflexiva (en activo) y usa al prototipado como manera de explorar y comunicar el abordaje a un problema difuso (Saikaly, 2005). Asumir el diseño como epistemología para abordar problemáticas en el marco de postconflicto, es también una invitación a politizar las acciones de diseño en la época actual, para actuar de manera activa en la generación de propuestas de tipo social (Bonsiepe,1985) que defiendan y garanticen el futuro de las nuevas generaciones y la posibilidad de una "Paz estable y duradera" producto de la creación y conservación de bienes comunes de todas y todos para todas y todos. Es así como la construcción del entramado social y político de un país constituye en sí mismo un problema de diseño.

En la investigación se propone un prototipo metodológico para analizar y crear iniciativas y comunidades de paz, el cual integra la teoría económica de Elinor Ostrom sobre los bienes comunes (2009), involucra además teorías del diseño que lo ubican a) en las dimensiones del poder y la emancipación como la propuesta por César González Ochoa (2015) b) lo humano y lo complejo como proponen Wolfgang Jonas (2004) y Offray Luna (2018) y c) el prototipo como hipótesis con la metodología diseño de investigación de Temud Leinonen (2014).

\section{Precisiones teóricas}

\section{La paz como un bien público}

En el proceso de estudio se hizo necesario el entendimiento del concepto de paz desde la perspectiva económica que permitiera acercamientos teóricos con la hipótesis plateada, sin bienes comunes no hay paz, para esto resultó pertinente la pregunta: ¿qué tipo de bien es la paz? La paz es un bien público y es deber 
del gobierno preservarla y mantenerla ${ }^{3}$; la paz requiere que todas las personas contribuyan, porque una vez lograda, todas podrán disfrutarla indistintamente del nivel de aporte. La paz es un bien público con alta extractibilidad y baja capacidad de exclusión (Pérez-Ducy, 2013, p. 199), es de tipo intangible y no se agota en la medida en que otros accedan a ella.

\section{Cuatro categorías para la gestión de recursos.}

\begin{tabular}{llll}
\hline & \multicolumn{2}{l}{ Sustractabilidad de uso } & \\
\hline & Alta & Baja \\
\cline { 2 - 4 } $\begin{array}{l}\text { Dificultad de excluir } \\
\text { beneficiarios potenciales }\end{array}$ & Fácil & $\begin{array}{l}\text { Recursos de uso común } \\
\text { (RUC): cuencas hidrológicas, } \\
\text { lagos, sistemas de irrigación, } \\
\text { pesquerías, bosques, etc. }\end{array}$ & $\begin{array}{l}\text { Bienes públicos: paz, seguridad de } \\
\text { una comunidad, defensa nacional, } \\
\text { conocimiento, protección contra } \\
\text { incendios, pronósticos del tiempo, } \\
\text { etc. }\end{array}$ \\
\cline { 2 - 4 } & Difícil & $\begin{array}{l}\text { Bienes privados: comida, ropa, } \\
\text { automóviles, vivienda etc. }\end{array}$ & $\begin{array}{l}\text { Bienes tarifa: cines, clubes } \\
\text { privados, guarderías, makerspace, } \\
\text { hackerspace. }\end{array}$ \\
\hline
\end{tabular}

Cuatro categorías para la gestión de recursos. Adaptado de Ostrom, 1990. (Ostrom, 2009, p. 22)

A pesar de ser un bien de responsabilidad gubernamental, la paz no puede ser entendida como un bien cerrado, ya que depende del sostenimiento de otros bienes y recursos que no son solo de tipo público. No es gratuito que el Acuerdo final de paz contemplara puntos relacionados con el uso de la tierra público-privada, bienes de tipo de RUC (recurso de uso común) como la delimitación de zonas de reserva forestal y bienes de tipo público como: el acceso a la democracia, la verdad, la memoria histórica y la justicia social.

Con este panorama, la teoría económica de Ostrom (2011) sobre la gobernanza de los bienes comunes, permitió en la investigación, comprender la importancia

\footnotetext{
${ }^{3}$ Artículo 22 de la Constitución Política de Colombia: La paz es un derecho y un deber de obligatorio cumplimiento. Involucra el poder para exigir al Estado y particulares el cumplimiento de obligaciones frente a la paz, y el derecho de estar protegido por el estado frente a la violencia y el terrorismo.
} 
de la descentralización de la gobernanza de la paz y plantear un prototipo metodológico de co-creación que involucrara personas e instituciones en el diseño de proyectos comunes con capacidad de crear y mantener bienes comunes. Se instaura la necesidad de pensar los procesos de gobernanza en la creación de diseños sociales para la paz, en este sentido los bienes comunes configuran una tercera rama en la variedad de posibilidades administrativas dentro de los procesos de gobierno. Con los bienes comunes las personas no son vistas solo desde el estrecho agujero del ciudadano como votante o la persona como cliente; los bienes comunes desde la perspectiva de Ostrom (2011) son vistos como una reconfiguración del poder distribuida, ya no en la dicotomía privado-público, sino expandida a una negociación con los sujetos frente a sus propios procesos de gobernanza, arguyendo que las personas pueden tomar decisiones activas y pueden ser supervisoras atentas de su cumplimiento.

\section{¿Desde qué epistemología del diseño abordar la paz?}

El proyecto de investigación transita diversas posturas teóricas alrededor del diseño para tender puentes con la teoría económica de los bienes comunes y sociopolítica alrededor de la paz y el conflicto; la perspectiva crítica de González Ochoa (2015) quien le atribuye al diseño un saber síntesis, posicionado en el interés emancipatorio, en las dimensiones del poder y de la teoría crítica ${ }^{4}$ desde una perspectiva habermasiana (Habermas, 1999). Posturas como la de Wolfgang Jonas (2004) y su teoría criticada por Luna Cárdenas (2018), afirman que el diseño es un saber en red y la teoría que se encarga de entender cómo esos saberes en red operan, es la teoría de sistemas sociales y la teoría de la evolución socio-cultural preocupada por la relaciones todo-parteconexiones. Allí se define que los saberes del diseño se sostienen a sí mismos

\footnotetext{
${ }^{4}$ La escuela de Frankfurt plantea la pugna teórica alrededor de la disputa entre la teoría crítica y la teoría tradicional, como reacción ante la unilateralidad hegemónica de la racionalidad teleológica (Cf. Max Weber). La teoría crítica de la sociedad se propuso interpretar y actualizar la teoría marxista originaria según su propio espíritu. Por ello, entiende que el conocimiento no es una simple reproducción conceptual de los datos objetivos de la realidad, sino una auténtica formación y constitución de estas (Wikipedia, 2017).
} 
y no se privilegia a ningún saber por encima; la teoría criticada de Wolfgang desde Luna, reafirma el carácter autopoiético del diseño, y en diálogo con las anteriores posturas teóricas, reivindica un posible puente entre la perspectiva crítica y emancipatoria "al desplazar el centro de lo social de lo comunicativo a lo humano, dando lugar a dichas perspectivas críticas y al diseñador como sujeto político" (Luna, 2018).

Al plantear el carácter autopoiético de la teoría del diseño, también se enfatiza el carácter del sujeto investigador; el diseñador como investigador se convierte en hacedor pero también en observador. El diseñador en tanto crea, también analiza y con esto se configura un proceso de retroalimentaciones constantes en donde existe un profundo involucramiento del sujeto con el objeto de investigación. Con respecto a esto Zimmermann definirá el ideal de diseñador como un pensador, "como alguien que no sólo diseña esto o aquello sino que toma una postura ética y política respecto de lo que significa, en concreto, un mejor mundo humano" (Universidad de Caldas, 2009, pp. 7-8).

\section{Metodología}

\section{El diseño basado en la investigación (DBI)}

El diseño de la paz se identificó como un problema complejo, que requiere de la participación de muchas personas e instituciones y sus propuestas de solución no son falsas ni verdaderas y suelen causar otros problemas (Leinonen, 2014). El Diseño basado en la investigación (DBI) propuesto por Teemu Leinonen (2010) propone el pensamiento de diseño como un enfoque alternativo para realizar investigaciones. El DBI fue el proceso metodológico con el cual se planteó una técnica basada en prototipos para evaluar, diseñar y acercar comunidades e iniciativas de paz en Colombia. 


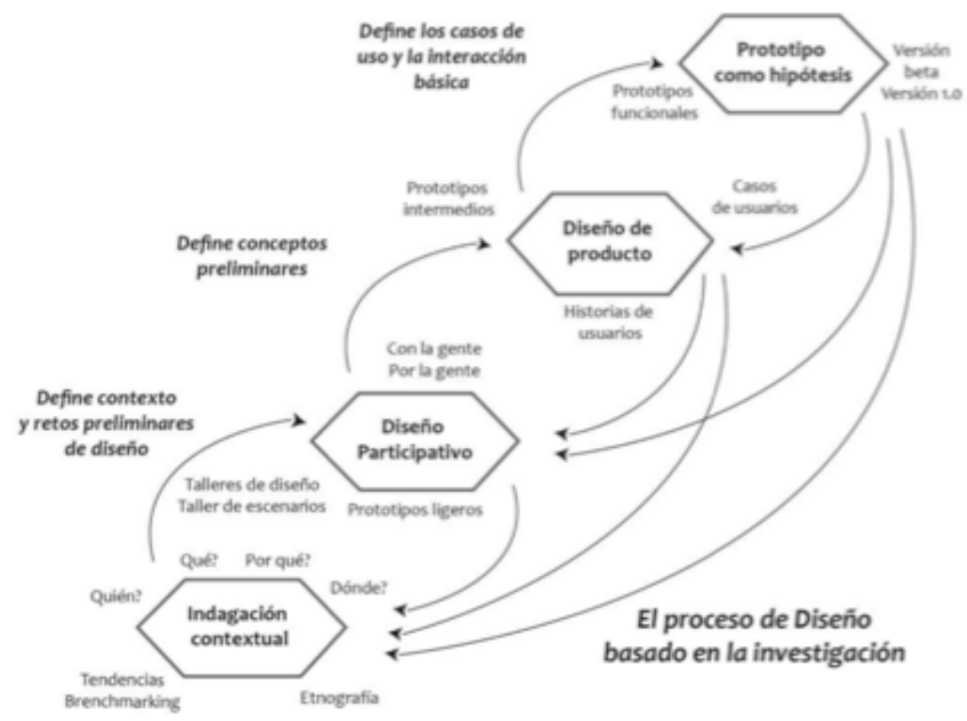

Diagrama 1 metodología DBI. Adaptación de Teemu Leinonen en Luna (2018).

El proceso de diseño basado en la investigación es una praxis de investigación inspirada en las teorías de Diseño (Ehn \& Kyng, 1987; Schön, 1987; Nelson \& Stolterman, 2003). Hace hincapié en las soluciones creativas, experimentos lúdicos y en la construcción de prototipos. Se alienta a los investigadores y diseñadores a probar diferentes ideas y conceptos. El proceso de diseño basado en la investigación se puede describir como un proceso continuo de definición y redefinición de los problemas y oportunidades de diseño, así como el diseño y re-diseño de prototipos. La mayoría de las actividades se realiza en un estrecho diálogo con la comunidad que previsiblemente utilizará las herramientas diseñadas. (Leinonen \& Durall Gazulla, 2014, p. 110) 
Las fases de diseño en este estudio son abordadas como procesos dinámicos en constante retroalimentación, lo que quiere decir que una fase puede apoyarse en la otra para confirmar o ampliar la información sobre lo desarrollado en cada una de estas. El estudio realizado abordó las fases del DBI de la siguiente manera:

\section{Indagación contextual}

El primer momento de la indagación se centra en la exploración del contexto socio-cultural en el cual está inmerso el diseño según Leinonen \& Durall Gazulla (2014, p. 110). La fase preliminar de diseño de prototipo, implicó un acercamiento a las comunidades y un accionar directo como investigadora partícipe de las comunidades indagadas (la Confluencia de Mujeres como proceso nacional y la Galería MakerSpace y Asmufare como comunidades regionales quindianas). El curso del Diplomado de Liderazgo para la Paz ofrecido por la Universidad Tecnológica de Pereira en el 2015, permitió recopilar la literatura necesaria para entender inicialmente los temas sobre conflicto en Colombia y procesos de paz en el mundo y plantear un primer ejercicio de diseño nombrado Transmediatones ${ }^{5}$. En esta fase se diseñan los formularios necesarios para la recopilación de la información dentro de dichas comunidades y se explora cómo éstas gestionan sus recursos desde la perspectiva de Elinor Ostrom, cómo dichas comunidades se consideran iniciativas de resistencia civil con los criterios de Hernández Delgado (2014) y en cuáles planes y programas del acuerdo de paz de la Habana se ubican o les interesa participar.

\section{Diseño participativo}

En la fase de diseño participativo lo más importante es el proceso de intervención de los involucrados en el problema de diseño, con ellos se discuten los resultados

\footnotetext{
${ }_{5}^{5}$ Para este diplomado se propone una transmediatón como proyecto final que intentaba "trasnmediar" los documentos producto de la Comisión Histórica del conflicto y sus Víctimas (CHCV) y otras dos transmediatones, en las cuales se trabajaron los comunicados conjuntos producto de los diálogos de La Habana. Para más información ingresar a http://visonte.org/publicaciones/transmediatones-del-conflicto
} 
producto de la primera fase en grupos pequeños de 4 a 6 personas para definir los prototipos ligeros. Los talleres planteados para esta fase están alrededor de ejercicios de prospectiva como la construcción de escenarios futuros o desafíos mentales de tipo "qué tal si". El involucramiento como sujeto-investigadoraparticipante posibilitó que la fase de diseño participativo se hiciera desde una relación directa con las comunidades; con esto se pudo explorar necesidades particulares dentro de los procesos comunitarios y las diferentes tensiones al interior de las acciones sociales. Esta fase fue desarrollada a través de prototipos mentales, talleres y actividades recurrentes dentro de los espacios en los cuales se participó, tales como encuentros nacionales con la Confluencia de Mujeres y creación del equipo base del departamento del Quindío y en la Galería Maker Space participante activa y colaboradora en los procesos de creación colectiva. Allí se recogen los deseos, dificultades y necesidades de dichas comunidades para plantear los prototipos.

\section{Diseño de producto}

Para Leinonen \& Durall Gazulla (2014, pp. 110-111) la fase de diseño de producto tienen como objetivo la construcción de los prototipos intermedios para la solución del problema de diseño. Allí el estudio y los investigadores especializados toman distancia de la comunidad para poder discutir cuestiones técnicas particulares en sus lenguajes específicos. En esta fase se toman decisiones de diseño, se analizan casos de usuarios y se desarrollan las propuestas que más adelante serán creadas como elementos funcionales.

Teniendo en cuenta las aproximaciones de las comunidades y los estudios teóricos realizados en este trabajo, para esta fase se realizan dos prototipos intermedios: el primero corresponde al prototipo metodológico para diseñar la paz como un bien común que más adelante será profundizado en este texto; el segundo prototipo corresponde a una herramienta llamada ComPaz ${ }^{6}$ 
presentada al Desafío Google 2017, con la cual las comunidades por medio de cartografías sociales, digitales y visualización de datos, podrán aplicar el prototipo metodológico, crear puentes con otras comunidades y visibilizar sus acciones.

ComPaz es resultado de dos tesis de investigación: "Diseño para la participación en el post-conflicto colombiano: cartografías para diseñar la paz desde los bienes comunes" (2018) de la Maestría en Diseño y Creación Interactiva presente en este documento y "Codiseñar autonomías: artefactos digitales amoldables, hacktivismo y ciudadanías" (2018) del Doctorado en Diseño y Creación del candidato Offray Vladimir Luna Cárdenas. ComPaz aborda la insuficiencia de mecanismos de participación incluyente y activa de la sociedad civil en marcos de conversaciones de paz con grupos armados. Está dirigida a comunidades e iniciativas creadoras de paz y bienes comunes. Es una plataforma abierta y colaborativa que permitirá visualización, diseño, articulación y evaluación de iniciativas desde la base, ayudando a su identificación y puesta en práctica.

La innovación del proyecto consiste en juntar tecnologías digitales, investigaciones teóricas, pensamiento en diseño, trabajo de campo y acciones prácticas, que funcionan de maneras dispersas, para el empoderamiento de comunidades de base. Las tecnologías usadas son: herramientas amoldables para visualización y narrativas de datos (Grafoscopio, Roassal, Pharo); las herramientas de georreferenciación (OpenStreetMap, Usahidi) que permiten agregar información desde distintos dispositivos, incluidos teléfonos celulares de bajo costo; los repositorios de código, datos y documentación distribuidos (DVCS: Distributed Version Control Systems, CKAN, Tupale) y sistemas de mensajería instantánea con cifrado punto a punto (Telegram, Signal); 
finalmente, están las "herramientas conceptuales" y desde el pensamiento en diseño que permite ubicar estas iniciativas geográficamente y temáticamente cercanas a los bienes comunes.

Con este prototipo se pretende crear comunidad tanto alrededor del desarrollo de la tecnología como la posibilidad de recolectar los datos y compartirlos de manera segura como insumo de investigación para la paz, debido a la naturaleza altamente replicable y de libre acceso de las plataformas y datos de código abierto. En esta fase de diseño de producto, se produjo un video ilustrativo ${ }^{7}$ para explicar ComPaz.

\section{Nuevamente diseño participativo}

La metodología vuelve a un diseño participativo con dos talleres comunitarios en los cuales se muestran los resultados de los diseños para que sean evaluados. Allí se realizan las observaciones pertinentes y los participantes de las comunidades manifiestan su interés en seguir evaluando y creando el prototipo presentado.

\section{El prototipo como hipótesis}

En la fase final metodológica se diseñan los prototipos funcionales que podrán ser probados y utilizados por personas en las condiciones cotidianas. Los resultados obtenidos en este momento todavía son considerados hipótesis ya que estos serán validados por los implicados directos del problema de diseño y allí será debatida la hipótesis. Tanto el prototipo metodológico como ComPaz están en proceso de búsqueda de financiación ${ }^{8}$ para establecerse como prototipos funcionales que puedan ser aplicados a otras comunidades e iniciativas distintas a con quiénes fueron diseñadas.

\footnotetext{
Video llustrativo ComPaz, véase en https://archive.org/details/compaz_201705\#

${ }^{8}$ Para colaborar con el proyecto ingresa a: http://visonte.org/
} 


\section{Dos metodologías que se transversalizan para la construcción de un prototipo metodológico para diseñar la paz desde los bienes comunes: Teemu Leinonen y Elinor Ostrom}

El DBI de Leinonen, aplicado como metodología de investigación desde el diseño para este estudio, también es utilizado en la creación del prototipo metodológico junto con el marco de análisis y desarrollo institucional (IAD) de Ostrom. Al hacer un análisis comparativo entre el Marco IAD de Ostrom y el DBI de Leinonen se identificó que el análisis del contexto se ubica como primer factor (diagnóstico de condiciones, atributos y reglas de la comunidad en Ostrom e indagación contextual en Leinonen), en una segunda instancia los procesos de acción (en Ostrom son llamados campos de acción y en Leinonen diseño tanto participativo como de producto), y en un tercer momento se ubica el análisis de resultados (en Ostrom Ilamados patrones de interacción, criterios de evaluación y resultados y en Leinonen el prototipo como una hipótesis que volverá al contexto para ser comprobada).

El prototipo metodológico desarrollado en el trabajo de investigación es una guía para la creación y mantenimiento de comunidades e iniciativas que estén alrededor del fortalecimiento y defensa de los bienes comunes. En esta guía se formulan tres momentos: mapearse, pensarse y repensarse. A continuación se presenta el diagrama del prototipo metodológico para diseñar la paz desde los bienes comunes propuesto en la investigación: 
METODOLOGÍA PARA DISEÑAR LA PAZ DESDE LOS BIENES COMUNES

MAPEARSE

Dónde

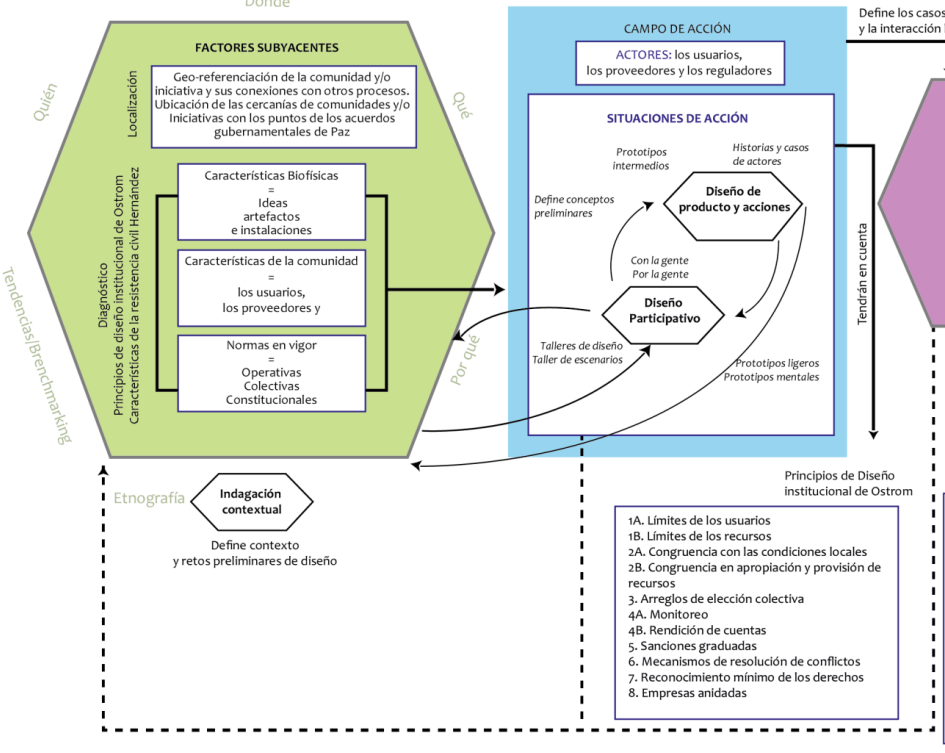

RE-PENSARSE

Diagrama 2. Integración Marco IAD de Ostrom y diseño de investigación de Temud Leinonen para diseñar la paz desde los bienes comunes. 


\section{Mapearse: indagación contextual, diagnóstico y ubicación}

El objetivo principal de este primer momento radica en la necesidad de las comunidades e iniciativas de hacer presencia, crear vínculos con otros procesos y ubicarse en los planes y programas construidos desde los procesos de paz realizados gubernamentalmente. Para este apartado la palabra "mapearse" tiene que ver con ubicarse en el espacio geográfico, ubicarse en el espacio temático y hacer un diagnóstico del estado en que se encuentran iniciativas y comunidades en cuanto a la cercanía o lejanía con el mantenimiento o creación de bienes comunes, esto quiere decir, analizar las características del contexto en el cual se mantienen, las ideas y los artefactos creados a partir de esas ideas, las características de las personas, las decisiones que toman dentro de la comunidad y un análisis de las normas que rigen los comportamientos dentro de ella.

\section{Pensarse: diseñar artefactos y acciones alrededor de la creación y el mantenimiento de bienes comunes}

Esta segunda fase está relacionada con el diseño de acciones (Arendt, 2005) para el auto-gobierno con metodologías creativas y pautas para el mantenimiento de bienes comunes. Son las intervenciones y decisiones adoptadas por las personas que involucran espacios, artefactos e ideas. ¿Qué permite este momento? A partir de talleres de arte y diseño, permite desplegar acciones de manera creativa y participativa dentro de las comunidades que no desconozcan las condiciones de cada contexto. Los actores involucrados en esta segunda fase son los usuarios, los proveedores y los reguladores de los bienes de las comunidades o los implicados en las iniciativas.

En Pensarse se abordan dos procesos de diseño principalmente: un diseño participativo que tiene que ver con las propuestas preliminares y prototipos 
ligeros que definen conceptos, desde las propuestas de los integrantes de la comunidad para abordar los problemas identificados en el diagnóstico. Aquí se proponen talleres como la creación de escenarios, taller de tipo Qué tal Sí entre otros. Seguido a esto se encuentra la creación de prototipos intermedios con el diseño de productos y acciones; esto se hace involucrando al personal más especializado de la comunidad, con intención de capacitarse y ahondar en el proceso de creación. Aquí se desarrollan productos y acciones que recojan lo que pasó en el diseño participativo y la indagación contextual. Las propuestas de diseño dentro de la comunidad estarán planteadas teniendo en cuenta los parámetros de diseño institucional (IAD) ${ }^{9}$ de Ostrom (2009). Es muy importante resaltar que el diseño de acciones puede manifestarse con la materialización por medio de artefactos. El resultado obtenido de esta fase no está cerrado y permite que sea constantemente reevaluado y puesto a prueba por la misma comunidad. En este momento es la comunidad quien valida el prototipo.

\section{Repensarse: evaluar las acciones diseñadas y su contribución a la generación de bienes comunes para la creación de paz dentro de la comunidad}

La etapa de "Repensarse" permite evaluar resultados obtenidos y patrones repetitivos en la interacción de los participantes de la comunidad. Se proponen algunos criterios de evaluación para examinar los patrones de interacción y los resultados obtenidos, aunque existen numerosos criterios potenciales de evaluación, en esta fase, después de haber creado un prototipo de comunidad ${ }^{10}$ o una iniciativa ${ }^{11}$ como hipótesis, se analiza bajo los patrones que aseguran que efectivamente ese diseño corresponde a una acción alrededor de los bienes

\footnotetext{
${ }^{9}$ 1A. Límites de los usuarios, 1B. Límites de los recursos, 2A. Congruencia con las condiciones locales, 2B. Apropiación y provisión, 3. Arreglos de elección colectiva, 4A. Monitoreo, 4B. Rendición de cuentas, 5. Sanciones graduadas, 6. Mecanismos de resolución de conflictos, 7 . Reconocimiento mínimo de los derechos, 8. Empresas anidadas.

${ }^{10}$ Se entiende como prototipo de comunidad, aquel grupo que al haber aplicado el proceso metodológico puede establecer sus propias reglas y los espacios y acciones necesarios para sostener los recursos que posibilitan el mantenimiento de dicho grupo de manera igualitaria y participativa. ${ }^{11}$ Aquí se entiende como iniciativa a proyectos, productos, sistemas productivos, artículos o procesos a los que se les ha aplicado la metodología para ser creados.
} 
comunes para generar paz. En el proceso de adaptación del Marco IAD y el DBI, para la creación de paz, los postulados de Hernández Delgado (2014) aportan fundamentalmente en lo que respecta a los criterios de evaluación pensando la paz desde la base y lo comunitario y aportando en todo el marco de la Investigación para la Paz. Los criterios mencionados en esta investigación son tomados desde su trabajo de tesis doctoral sobre algunos de los factores más relevantes que permanecen en las nombradas comunidades de Paz en Colombia y otros criterios que tienen que ver con los bienes comunes del conocimiento planteados desde Ostrom y Hess (2016).

Criterios para evaluar la paz perfectible: debido al amplio alcance de estos criterios y las implicaciones de su estudio en términos de tiempo y financiación, dichos criterios, a diferencia de los otros momentos del prototipo metodológico, han sido formulados como recuento teórico y todavía no se han desarrollado los instrumentos ni puesto a prueba en la práctica comunitaria de este estudio. Los criterios son: incremento de ambientes sin violencia, sostenibilidad y conservación de los recursos, estándares de participación e igualdad, eficiencia económica, la equidad mediante la equivalencia económica, equidad redistributiva, capacidad para aceptar otros modelos de gobernanza y aceptar la naturaleza humana, capacidad para aceptar la contingencia del prototipo, despliegue de creatividad e imaginación, empoderamiento, confianza.

\section{Resultados}

\section{Hallazgos del análisis del prototipo metodológico para diseñar la paz desde los bienes comunes}

Las metodologías en diseño puestas en marcha en el estudio permitieron una forma particular de abordar las problemáticas en donde las soluciones son prototipos perfectibles e inacabados y de naturaleza necesariamente contextual. 
Esto proporciona una manera de abordar las propuestas de paz pensadas como elementos en proceso, perfectibles e inacabados donde las comunidades se reinventa a sí misma dependiendo de sus contextos y momentos históricos particulares. El prototipo metodológico resulta pertinente porque permite a las comunidades analizar situaciones de su entorno y la normas que preexisten en él, pero también invita a la creación de nuevas normas, nuevos artefactos y nuevas acciones dentro de éstas, otorgando un carácter no estático sino dinámico y perfectible de realidades y contextos; con esto el proceso creativo se reconoce de vital importancia para mantener las relaciones de gobernanza, auto-gestionadas, autónomas y horizontales.

En cuanto a situaciones internas las comunidades pudieron entenderse como artefactos perfectibles y en construcción constante, lo cual permite la reevaluación y autocrítica de las acciones internas, teniendo en cuenta las contingencias del entorno tanto medio ambiental como sociopolítico.

Las comunidades con las que se aplicó el prototipo metodológico se nombraron como iniciativas de resistencia civil, esto permitió establecer una diferencia entre los procesos de gobernabilidad versus los procesos de gobernanza; teniendo el primero como la construcción del poder y la toma de decisiones entre los actores visibles de las comunidades como líderes o representante del Estado, mientras en el segundo se entiende como la toma de decisiones y poder de todos y todas, para todos y todas desde la base, sin exclusión de personas ni ideas. Con respecto a esto, sobre todo en lo que concierne a la gobernanza, se analiza de manera crítica el concepto, el cual nace en el seno de la economía capitalista para librar de responsabilidad al gobierno y atribuirles responsabilidades a los ciudadanos.

La gobernanza se convierte en un arma de doble filo para las comunidades si no es entendida desde una ciencia política crítica. Un hallazgo importante 
de corte teórico, el cual aportará a las implementaciones futuras de este estudio, es el de Bob Jessop (2008), quien invita a la metagobernanza. Este autor reseña el concepto de gobernanza desde un Estado de matriz marxista pero que ha ido evolucionando incorporando elementos de la teoría de la regulación, los sistemas autopoiéticos, las teorías del discurso y de los análisis de Gramsci y Poulantzas. La propuesta para superar la trampa puesta por la dualidad totalizante estado-mercado con la gobernanza, es el sistema de comarcas, en la cual, mientras los ciudadanos cooperan para la construcción de Estado, también reclaman las responsabilidades conseguidas con luchas sobre un Estado social de derecho. En el sistema de comarcas $^{12}$ se organiza así: una comarca mínima que garantice los mínimos vitales y los derechos fundamentales, una comarca democrática con sistemas de participación sólidos, una comarca femenina que reivindique a la mujer, establezca paridad e instaure una comprensión feminista del mundo, una comarca endeudada en la cual se niegue la deuda externa, opresora e impagable sobre todo en los países latinoamericanos, una comarca ecológica en la cual se defiende la naturaleza, una comarca universitaria, una comarca social, una comarca informada, una comarca obrera y por último una comarca multicultural (Jessop, 2008). Si bien dentro de las comunidades de base es necesario el entendimiento de los sistemas administrativos y económicos y la capacidad de estas de autogobierno, no puede haber un alejamiento en la defensa del Estado social de derecho y la exigencia de responsabilidades al gobierno por medio de la protesta, la resistencia, la acción y la participación.

El prototipo metodológico corresponde a una guía para la formación de comunidades "innovadoras"; esto quiere decir, comunidades que dentro de sí mismas tengan el poder para crearse y compartir sus recursos de tal manera

\footnotetext{
12 Muchas colectividades, si bien no se entienden a sí mismas desde las comarcas de Jessop, dialogan fuertemente con esta idea. Podría ser entendido como sistema de comarcas a las comunidades hacker/maker que deconstruyen el poder embebido en la técnica desde el quehacer. El hecho de que existan colectividades en diálogo con esas comarcas también permite ver los lugares de cruces entre ellas como los feminismos de datos, eco/bio-makers/hackers.
} 
que todos puedan ser partícipes tanto de los beneficios como de las acciones para sostenerlos y mantenerlos. En cuanto a los procesos de participación, mediante el prototipo se identificó la importancia de los niveles de participación comunitaria, para establecer que no solo tiene acceso a los recursos de una comunidad quien participa de la actividad para conseguirlo, sino también cuyas personas está trabajando en otras actividades, en beneficio colectivo.

Las comunidades por medio de esta guía pudieron crear acciones de manera modular; la solución a los problemas planteados no resolvía el todo sino las partes, las cuales en adición iban acercándose a la solución del todo. Un ejemplo, en la Galería Maker Space se propuso el prototipo metodológico para ser utilizado para la Feria Galembera y la construcción de los estatutos de la Corporación La Galería Maker Space, para abordar los problemas de financiación y organización; en la Confluencia de Mujeres está siendo utilizado para los procesos de creación de plataformas de comunicación popular, feminista y comunitaria, en respuesta al problema de construcción de planes estratégicos para la acción política de las mujeres en Colombia y en Asmufare para la creación de la identidad visual y las acciones para enfrentar las amenazas de desalojo, identificando como problema amplio el desplazamiento y la brecha existente en Colombia entre el campo y la ciudad. Algunos de los prototipos como hipótesis que surgieron dentro de las comunidades, posiblemente no cuentan con los patrones estéticos de diseño en el mercado. Los prototipos que han ido surgiendo dentro de las comunidades han sido pertinentes para ellas y se han comprendido como "diseños a la medida" de sus contextos y necesidades.

\section{Hallazgos del análisis en cuanto a los puentes del diseño como transdisciplina y sus aportes para hacer investigación para la paz}

La paz es un bien público, el cual debe ser asegurado por el gobierno en representación del presidente, sin embargo es co-creada en colectivo con 
la sociedad civil y depende de la capacidad que tenga ésta de agruparse y organizarse para construir y mantener, desde sus cotidianidades bienes para todos y todas, en consecuencia bienes comunes. El DBI de Leinonen conjugado con el marco IAD para la construcción de bienes comunes de Ostrom, permite el acercamiento de la inteligencia proyectual del diseño a las comunidades no especializadas, ya que posibilita un trabajo conjunto entre implicados y contexto e instaura capacidad en el desarrollo de prototipos para solucionar problemáticas sociales. Con esta metodología la práctica del diseño es horizontal y participativa e involucra necesariamente la capacidad antropológica proyectual del ser humano de crear las herramientas necesarias para la solución de los conflictos particulares de su entorno.

Haber identificado que la paz no es un objeto concreto, sino que tiene que ver con las diversas acciones del cotidiano y la cultura, posibilitó comprender que no hay proyectos de paz sino acciones que posibilitan relaciones sociales pacíficas. El proceso de (de)construcción de los mundos posibles planteado en las teorías del diseño, posibilita pensarse las acciones de paz como un camino de creación que procure una estética y una belleza de los artefactos y acciones creadas por humanos, como acción política. Al respecto, el fortalecimiento y entendimiento de los bienes comunes posibilitó ambientes de gobernanza dentro de las comunidades en donde sus integrantes fueron conscientes de la necesidad de luchar contra los problemas de despojo, privatización, exclusión de recursos, cooptación y desarraigo que han dado origen al conflicto en el país.

La politización de las acciones de diseño propuestas en el estudio, esperanzan a las personas hacia una voluntad de poder, es decir la capacidad que tienen las comunidades de cambiar sus propias realidades por medio del pensamiento proyectivo (Aicher, 1994). Identificar que el diseño, al oscilar entre la praxis y la técnica (Ochoa, 2015) proporciona una manera de construir poder, tendiendo puentes entre teorías de diseño, teorías económicas y teorías sociopolíticas. 
Se identificó el diálogo, la esperanza y la creatividad como características necesarias para la construcción de gobernanza; estas acciones tejen lazos sociales dentro de las comunidades. El diálogo es por excelencia el mecanismo de resolución de conflicto más reiterado en las comunidades debido a su eficiencia y a los bajos costos que implica.

Sin bienes comunes no hay paz, fue la hipótesis planteada en esta investigación, responder su carácter de falso o verdadero contradice lo que expone Jonas (2004), quien manifiesta que en la investigación en diseño no se buscan verdades absolutas sino encontrar el carácter plausible de la hipótesis, en este sentido esta investigación al indicar que la paz es un proceso perfectible e inacabado, también propone su hipótesis como plausible.

\section{Recomendaciones generales analizadas en el proceso de investigación}

La creación de metodologías, categorizaciones y estandarizaciones en el abordaje de estudios sociales para procurar dar orden y practicidad al estudio, tienen el riesgo de volverse estáticos lineales y rígidos; racionalizar de manera extrema la intervención y el trabajo con las comunidades puede desconocer procesos simbólicos, antropológicos y cosmogonías propias de éstas. Para minimizar este riesgo, es importante resaltar que aunque la propuesta es un marco, se debe procurar por realizar un proceso de intervención flexible que permita a las comunidades crear a partir de sus creencias, símbolos y formas particulares de acercarse a las relaciones con otros y la naturaleza desde sus cosmovisiones. Este estudio de las comunidades desde el pensamiento en diseño, debe procurar, en su mayor medida, involucrar de manera más profunda estudios antropológicos, feministas y simbólicos que nazcan dentro de las comunidades. Los prototipos resultados del estudio, parten de una visión académica con influencia de teorías occidentales; posiblemente para otras culturas, la teoría de los bienes comunes no sea asumida, sin embargo, en este 
trabajo se plantea un prototipo metodológico que puede entrar en diálogo y ser adaptado en las comunidades, teniendo en cuenta las cosmovisiones que aquí no han sido contempladas.

La figura de la persona experta para la aplicación de esta propuesta es necesaria, debido a que se evidenció la necesidad de apoyo a las comunidades en forma de posibilitadoras desde una mirada externa, que logre hacerles preguntas suficientemente sólidas para que en el núcleo de las comunidades puedan ser respondidas. La persona experta para el acompañamiento de la implementación del prototipo, debe respetar de manera considerable las decisiones de la comunidad y tratar de adaptar el marco a aquellas posturas y soluciones como sujeto posibilitador y no dominador.

Diseñar la paz corresponde a la capacidad de una sociedad para la participación y propuestas de accionar político en la creación de recursos amplios y de beneficios colectivos (Galtung, 1985), con esto se identifica que una de las principales características para que una comunidad se auto-organice, que trabaje de forma colectiva y participe, es la esperanza en el futuro, la comunidad en la cual este punto era crítico, manifestó la mayor dificultad en la participación.

Finalmente, se recomienda un énfasis en la política pública y el uso de tecnología y manejo de datos para la intervención en procesos de paz. Con esto en primera medida es necesario el desarrollo de políticas públicas que fomenten la cooperación y no la creación de incentivos para egoístas racionales (Ostrom, 2008) (personas que actúan para maximizar el interés propio), con esto las comunidades que están organizadas alrededor de la conservación y creación de bienes comunes deberían ser visibles, apoyadas estatalmente y estudiadas de manera más rigurosa y profunda en el ámbito académico. En cuanto al uso de tecnología es necesario el uso y conservación de herramientas 
contrahegemónicas ${ }^{13}$ que aseguren que los datos sean bienes comunes, seguros y abiertos para permitir investigaciones futuras alrededor de la paz. El hecho de que sea abierto no quiere decir que sea inseguro, contrario a esto, procurar que los datos sean abiertos posibilita la autonomía de las comunidades frente a lo que quieren compartir y lo que no dentro de las plataformas digitales. Es necesario que las implementaciones de acciones sociales en la cuales se busca fortalecer bienes comunes también sean creadas con herramientas tecnológicas que estén en coherencia filosófica ${ }^{14}$.

En recuento, este documento recoge de manera amplia el proceso de investigación en diseño el cual propone - a manera de prototipo- una técnica generadora de participación ciudadana para la construcción de paz. Esta propuesta aporta un marco metodológico, que desde el pensamiento en diseño y su forma particular de abordar las problemáticas —se piensan las soluciones como prototipos perfectibles e inacabados y de naturaleza necesariamente contextual_ brinda una manera de diseñar propuestas de paz como elementos en proceso, perfectibles e inacabados, donde las comunidades se reinventan a sí mismas, ajustan soluciones a sus contextos y momentos históricos particulares proporcionando un insumo para hacer investigación para la paz.

\footnotetext{
${ }^{13} \mathrm{GNU}$, Datos abiertos, licencias abiertas, hardware abierto.

${ }^{14} \mathrm{Al}$ igual que las herramientas tecnológicas, la publicación científica que circula en revistas indexadas y que plantea la necesidad de proteger y crear bienes comunes, debe tener coherencia filosófica y política. Por tal motivo, el ejercicio de escritura de este artículo no se acaba en la academia, ni es exclusivo para ella, y la publicación de documentos que tienen que ver con el tema y dan cuenta de la investigación mencionada, circulan por los canales de internet y por las organizaciones que están participando en la conservación de bienes comunes del conocimiento y ciencia abierta.
} 


\section{Referencias}

Aicher, O. (1994). El mundo como proyecto. Barcelona: Gustavo Gili.

Arendt, H. (2005). La condición humana (1. a ed.). Buenos Aires: Paidós.

Bonsiepe, G. (1985). El diseño de la periferia. Debates y experiencias. México, D.F.: Gustavo Gili México.

Castrillón Arango, A. (2018). Diseño para la participación de comunidades e iniciativas en el post-conflicto colombiano: cartografías para diseñar la paz desde los bienes comunes. Tesis de maestría. Universidad de Caldas. Manizales. Colombia. Recuperado de: http://visonte.org/repos.fossil/adriana/uv/maestria/ tesis_final_version_publicar.pdf

Galtung, J. (1985). Sobre la paz. Barcelona: Fontamara.

González Ochoa, C. (2015). El diseño como acción. Hacia una ética de la actividad proyectual. (1. ${ }^{\text {a }}$ ed.). Manizales: Universidad de Caldas.

Habermas, J. (1999). Teoría de la acción comunicativa. Madrid, España: Taurus.

Hernández Delgado, E. (2014). Empoderamiento pacifista de experiencias comunitarias locales en Colombia (tesis doctoral). Universidad De Granada Instituto de la Paz y los Conflictos Granada. Recuperado de http://hera.ugr.es/ tesisugr/24287106.pdf

Jesob, B. (2008). El futuro del Estado capitalista. Madrid: Catarata.

Jonas, W., \& Meyer, J. (2004). Design theories and processes are evolutionary artifacts. In Mind The Gap. On knowing and not-knowing in design. Alemania: Hauschild Verlag / University of the Arts Bremen.

Leinonen, T., y Durall, E. (2014). Pensamiento de diseño y aprendizaje colaborativo. Comunicar, 21(42), 107-116. https://doi.org/10.3916/C42-2014-10

Luna, O.V. (2014). Ecología de saberes en diseño: Un ejemplo desde los discursos autopoiéticos. Presentado en examen de candidatura para acceder al título de 
Doctor en Diseño y Creación, Universidad de Caldas Manizales Colombia. Recuperado de http://bit.ly/2zUdjtl

Luna, O.V. (2018). Codiseñar autonomías: artefactos digitales amoldables, hacktivismo y ciudadanías (tesis doctoral). Universidad de Caldas. Bogotá D.C. Colombia. Recuperado de https://mutabit.com/repos.fossil/doctorado-offray/ uv/Tesis/Escrito/Textolntegrado/tesis.pdf

Ostrom, E. (2011). El Gobierno de los Bienes Comunes. la evolución de las instituciones de acción colectiva (2. ${ }^{a}$ ed.). México: Fondo de Cultura Económica, Universidad Autónoma de México, Centro Regional de Investigaciones Multidisciplinarias.

Ostrom, E. y Hess, C. (2016). Un marco de análisis de los Bienes Comunes del conocimiento. En Los bienes comunes del conocimiento. Madrid, España: Traficante de sueños.

Ostrom, E. (2009). Más allá de los mercados y los Estados: gobernanza policéntrica de sistemas económicos complejos. Presentado en Conferencia de recepción del premio Nobel de Economía, Estocolmo. Recuperado de http://www.scielo. org.mx/pdf/rms/v76nspe/v76nspea2.pdf

Pérez-Ducy, E. (2013). Coproducción de la paz social: la elevación de las penas para adolescentes vistas según las teorías de Elinor Ostrom. Ciencia y Sociedad [en línea], 38, 195-214.

Saikaly, F. (2005). Approaches to Design Research: Towards the Designerly Way. In 6th International Conference of the European Academy of Design, Design System Evolution. University of the Arts Bremen.

Universidad de Caldas. (2009). Doctorado en Diseño y Creación. Manizales, Colombia: U de Caldas. Recuperado de http://bit.ly/2iwaZS3

Como citar: Castrillón, A. (2020). Diseño para la participación de comunidades e iniciativas en el postconflicto colombiano: cartografías para diseñar la paz desde los bienes comunes. Revista KEPES, 17 (21), 335-360. DOI: 10.17151/kepes.2020.17.21.12 